\section{Confiabilidade das informações das declarações de nascido vivo com registro de defeitos congênitos no Município do Rio de Janeiro, Brasil, 2004}

\author{
Reliability of birth defect data on birth \\ certificates of Rio de Janeiro, Brazil, 2004
}

Fernando Antônio Ramos Guerra 1

Juan Clinton Llerena Jr. 1

Silvana Granado Nogueira da Gama 2

Cynthia Braga da Cunha 2

Mariza Miranda Theme Filha 3

\footnotetext{
1 Instituto Fernandes

Figueira, Fundação Oswaldo

Cruz, Rio de Janeiro, Brasil.

2 Escola Nacional de Saúde

Pública Sergio Arouca,

Fundação Oswaldo Cruz,

Rio de Janeiro, Brasil.

3 Superintendência de

Vigilância em Saúde,

Secretaria Municipal de

Saúde do Rio de Janeiro,

Rio de Janeiro, Brasil.

Correspondência

F. A. R. Guerra

Departamento de Obstetrícia,

Instituto Fernandes Figueira,

Fundação Oswaldo Cruz.

Av. Oswaldo Cruz 87,

apto. 101, Rio de Janeiro, $R J$

22260-060, Brasil.

fguerra@iff.fiocruz.br
}

\section{Abstract}

This study assessed the reliability of birth certificate data related to birth defects in Brazil's Live Birth Information System (SINASC). We selected 24 maternity hospitals in the Unified National Health System (SUS) and compared the reports of birth defects from birth certificates with medical records of mothers and live born infants in the city of Rio de Janeiro for the year 2004. After transposing the data to a specific form, the birth defects were coded by types and organ systems and compared to the SINASC data. The most commonly affected organs involved the central nervous and musculoskeletal systems. Agreement was more than 50\% for the digestive, genitourinary, and musculoskeletal systems and chromosomal anomalies. Prevalence-adjusted kappa varied according to 2 or 3-digit ICD-10 analysis, with better results for the musculoskeletal, digestive, and genitourinary systems and congenital anomalies, and worse for the central nervous and cardio-circulatory systems, eye, neck, and ear malformations, and cleft lip and palate. The results were unsatisfactory, suggesting the need for more investments to train the persons responsible for completing birth certificates in maternity hospitals and develop a model for coding birth defects on these documents.

Abnormalities; Birth Certificates; Information Systems

\section{Introdução}

Os defeitos congênitos ocorrem em cerca de $2 \%$ a 3\% dos recém-nascidos e são os principais responsáveis pela mortalidade infantil em nações desenvolvidas 1. Também na América Latina, essas patologias têm contribuído significativamente para a mortalidade de menores de um ano 1,2,3 e no Brasil, embora nos últimos anos o número de óbitos nesta faixa etária decorrentes de grande parte das doenças tenha declinado, o mesmo não se verifica com aqueles relacionados aos defeitos congênitos, hoje a segunda causa de mortalidade infantil no país 4 . Acredita-se que a prevalência global de anomalias cromossômicas e malformações congênitas nos países em desenvolvimento seja maior que em nações industrializadas e sistematicamente subestimadas devido à deficiência de informações 5 .

A escassez de dados oficiais no Brasil referentes aos defeitos congênitos aponta para a necessidade do aperfeiçoamento dos sistemas de informação já existentes. Em 1999, tornou-se possível o relato destas informações nas declarações de nascido vivo por meio da introdução do campo 34, específico para o registro destas patologias. Este novo modelo passou a ser usado efetivamente no Município do Rio de Janeiro a partir de 2000.

Em todo o mundo, os dados provenientes dos certificados de nascimento são importantes na construção dos indicadores de qualidade em 
saúde e para a formulação de políticas de assistência. Numerosos estudos sobre a qualidade das informações por eles geradas estão hoje disponíveis na literatura científica $6,7,8$. No Brasil, a declaração de nascido vivo é o instrumento de coleta de dados do Sistema de Informações sobre Nascidos Vivos (SINASC) e caracteriza-se por ser universal (uma declaração por criança), padronizada e de baixo custo. Os dados vitais por ela gerados fornecem volumosas informações para estudos epidemiológicos e bases para planejamento e atividades de prevenção, assim como políticas na área da saúde materno-infantil 9,10.

Os estudos realizados sobre o SINASC versam na maioria sobre a sua implantação, cobertura e caracterização dos nascidos vivos 9,10,11,12,13. Aqueles que tratam sobre a qualidade das informações apontam para a baixa confiabilidade de alguns campos da declaração de nascido vivo e recomendam maior atenção no preenchimento do instrumento e conseqüentemente maior controle de qualidade 14,15 .

Ao nos referirmos especificamente aos defeitos congênitos, pouco se sabe sobre a qualidade destas informações; bem diferente de países europeus ou da América do Norte, onde esta variável encontra-se incluída nos certificados de nascimento há mais de vinte anos. Nestes países, a existência de programas regionais e nacionais de vigilância de defeitos congênitos serve ainda como padrão ouro para estudos de avaliação de qualidade das informações constantes nos certificados 16,17,18. Embora um estudo publicado recentemente sobre a confiabilidade de dados do SINASC no Município do Rio de Janeiro tenha demonstrado um excelente índice de concordância quanto à presença de defeitos congênitos 15 não se verificou se o mesmo se repete quanto ao tipo de defeitos congênitos relatado na declaração de nascido vivo.

Este artigo tem como objetivo avaliar a confiabilidade das informações contidas nas declarações de nascido vivo em 2004 no Município do Rio de Janeiro, segundo a presença e o tipo de defeitos congênitos registrado, assim como de algumas variáveis relativas a estes recém nascidos e suas mães.

\section{Métodos}

Realizou-se nesta pesquisa uma análise da confiabilidade das informações sobre defeitos congênitos contidas nas declarações de nascido vivo geradas nas instituições militares e do Sistema Único de Saúde (SUS) do Município do Rio de Janeiro no período de 10 de janeiro a 31 de dezembro de 2004. Consideraram-se como defeitos congênitos para fins descritivos, além das malformações, as anomalias cromossômicas.

A declaração de nascido vivo é o formulário de coleta de dados do SINASC, que deve ser preenchido para todos os nascidos vivos nas maternidades do país. Seu preenchimento é feito por funcionários administrativos, enfermeiros ou médicos sendo recomendado que qualquer defeito congênito detectado no exame do recémnascido deve ser descrito sumariamente no campo especificado. No Rio de Janeiro, a codificação dos defeitos congênitos é realizada nas Coordenações de Áreas Programáticas (CAP) sob supervisão da Secretaria Municipal de Saúde do Rio de Janeiro (SMS-RJ), com base na Classificação Estatística Internacional de Doenças e Problemas Relacionados à Saúde, décima revisão (CID-10) 19, por digitadores treinados pela própria SMS-RJ.

Todas as maternidades do Município do Rio de Janeiro foram convidadas a participar do estudo, porém as maternidades privadas não disponibilizaram os prontuários dos pacientes durante o período definido para coleta de dados, o que representou 15,8\% dos recém-nascidos com defeitos congênitos em 2004. Contabilizaram-se, portanto, 24 instituições das quais noves eram municipais, três estaduais, três federais, três universitárias, cinco militares, duas filantrópicas e uma privada conveniada com o SUS. Foram separadas 761 declarações de nascido vivo com o campo 34 (referente à presença de defeitos congênitos) preenchido positivamente.

Procedeu-se, após contato prévio e autorização formal da direção de cada instituição selecionada, à busca dos prontuários das 761 mães e de seus respectivos filhos nos arquivos hospitalares. A coleta de dados foi realizada pelo pesquisador principal e duas médicas especialistas em medicina fetal previamente treinadas, utilizando-se formulários de coleta elaborados com esta finalidade específica. Estes formulários constavam do número da declaração de nascido vivo e de variáveis referentes à mãe, à gestação, ao parto e ao recém-nascido, ou seja, idade, estado civil, escolaridade, número de filhos vivos e mortos em gestações anteriores, duração e tipo da gravidez (única ou múltipla), número de consultas pré-natais, tipo de parto, sexo, índice de Apgar, peso ao nascer, presença e tipo de defeito congênito. A duração da gestação foi obtida por cálculo baseado na data da última menstruação segundo a regra de Nagele 20 ou pela ultra-sonografia obstétrica e expressa em semanas.

Todas as informações referentes aos defeitos congênitos foram descritas e codificadas segundo a CID-10 pelos mesmos profissionais que realizaram a coleta, com especificação de até três dígitos e posteriormente agrupadas por apare- 
lhos e sistemas orgânicos. Os critérios utilizados para a codificação obedeceram rigorosamente às descrições diagnósticas encontradas nos prontuários, utilizando-se um código para cada defeito congênito encontrado. Aos pesquisadores foi orientado procurarem nos prontuários todos os diagnósticos de defeitos congênitos realizados ou confirmados após o nascimento e durante a internação do recém-nascido. Ressalta-se que na declaração de nascido vivo, mesmo que vários defeitos congênitos estejam descritos, é permitido codificar somente um deles. Todas as informações colhidas nos questionários foram posteriormente digitadas em banco de dados criado com esta finalidade.

A análise de concordância foi realizada com base na comparação entre os dados da internação registrados no prontuário hospitalar e das informações contidas na declaração de nascido vivo, tendo como referência as anotações nos prontuários. Para esta análise criou-se um novo banco com o pareamento dos dados obtidos em cada uma das fontes. O identificador empregado no pareamento foi o número da declaração de nascido vivo.

Como medidas de confiabilidade utilizaramse o percentual de concordância, calculado através da divisão da soma da diagonal da tabela pelo total de casos; os índices kappa simples para as variáveis nominais e ordinais (KS), kappa ponderado para as ordinais (KP), kappa ajustado pela prevalência (KPA) para as variáveis nominais e ordinais com o objetivo de minorar o efeito de prevalência no KS 21 e o coeficiente de correlação intraclasse (CCI) para as contínuas.

$\mathrm{O}$ índice kappa compara a diferença entre as concordâncias observada e esperada chegando ao valor máximo possível para esta diferença. Estas estatísticas podem variar entre -1 (concordância observada menor do que a esperada ao acaso) e 1 (concordância perfeita) 22. Utilizaramse, para a interpretação dos valores de kappa, os critérios de Landis \& Koch: concordância pobre (menor que 0); discreta $(0-0,20)$; razoável $(0,21$ $0,40)$; moderada $(0,41-0,60)$; substancial $(0,61$ $0,80)$ e quase perfeita $(0,81-1,00) 23$. O intervalo de confiança adotado foi de $95 \%$ onde se testou a hipótese nula de que a estatística de concordância estimada seria igual a zero.

Foi avaliada a confiabilidade da variável diagnóstico do defeito congênito - segundo a CID-10, capítulo XVII (Q00-Q99), com 2 e 3 dígitos por sistemas e aparelhos comprometidos -, assim como aquelas referentes às mães e suas gestações (idade, estado civil, escolaridade, número de filhos vivos e mortos em gestações anteriores tipo de gravidez, número de consultas de prénatal, duração da gestação, tipo de parto) e ao recém-nascido (Apgar no lo e 5o minuto e peso ao nascer).

O programa estatístico utilizado foi o WINPEPI com o uso do componente PAIRS 24. $\mathrm{O}$ estudo foi submetido à análise e aprovado pelo Comitê de Ética em Pesquisa do Instituto Fernandes Figueira (IFF) da Fundação Oswaldo Cruz (FIOCRUZ).

\section{Resultados}

No ano de 2004, houve segundo o SINASC, um total de 903 recém-nascidos com defeitos congênitos representando $1,1 \%$ de um total de 85.983 nascidos vivos no Município do Rio de Janeiro, excluindo-se 8.869 nascimentos com informação ignorada. Dos 903 casos foram selecionados 761 que ocorreram nos estabelecimentos integrantes do SUS além das instituições militares e destes 761, conseguiu-se obter dados de 680 prontuários, correspondendo a uma perda de 10,6\%. Dos 680 nascimentos, 29 não apresentavam defeitos congênitos sendo excluídos da base final que por fim foi composta de 651 casos.

Procedeu-se à comparação entre os 81 recémnascidos considerados como perdas e os casos incluídos no estudo (651), utilizando-se algumas informações disponíveis no banco de dados do SINASC. Não se observou diferença significativa entre os dois grupos pelo teste $\chi^{2}$ de diferença de proporções no que se refere ao sexo e peso do recém-nascido, escolaridade e faixa etária maternas e do número de consultas pré-natais.

A Tabela 1 apresenta as estimativas do kappa simples, kappa ajustado pela prevalência, o número de nascidos vivos com defeitos congênitos além dos percentuais de concordância segundo aparelhos e sistemas acometidos por defeitos congênitos e classificados pela CID-10 para 2 e 3 dígitos. Deve-se ressaltar que o resultado da análise de confiabilidade para 2 e 3 dígitos da CID-10 relaciona-se com o grau de especificidade do diagnóstico do defeito congênito. Os percentuais de informações ignoradas foram no geral inexistentes exceto para os defeitos congênitos do aparelho digestivo e do pé com 5,6\% e $1,4 \%$ respectivamente. Observou-se, segundo os prontuários, uma maior freqüência de nascimentos com defeitos congênitos classificados como do aparelho osteomuscular (nascidos vivos $=285$ ) e do sistema nervoso central (nascidos vivos $=129$ ). Sistematicamente, os percentuais de concordância para os agrupamentos em dois dígitos foram maiores quando comparados ao agrupamento de três dígitos. Os maiores percentuais de concordância (acima de 50\%) para 2 dígitos da CID-10 encontraram-se naqueles casos 
Medidas de confiabilidade das informações sobre defeitos congênitos segundo sistemas e aparelhos comprometidos. Município do Rio de Janeiro, Brasil, 2004

\begin{tabular}{|c|c|c|c|c|c|}
\hline Variáveis & KS & KAP & Nascidos vivos & $\%$ ignorados & \% concordância \\
\hline \multicolumn{6}{|c|}{ Sistema nervoso central (Q00-Q07) } \\
\hline Dois dígitos & $0,36(0,27-0,45)$ & 0,42 & 129 & 0,0 & 45,0 \\
\hline Três dígitos & $0,24(0,17-0,32)$ & 0,28 & & & 29,5 \\
\hline \multicolumn{6}{|c|}{ Olhos, face, orelha e pescoço (Q10-Q18) } \\
\hline Dois dígitos & $0,26(0,05-0,46)$ & 0,38 & 21 & 0,0 & 42,9 \\
\hline Três dígitos & $0,16(0,01-0,31)$ & 0,15 & & & 19,0 \\
\hline \multicolumn{6}{|c|}{ Coração e aparelho circulatório (Q20-Q28) } \\
\hline Dois dígitos & $0,32(0,11-0,53)$ & 0,35 & 17 & 0,0 & 41,2 \\
\hline Três dígitos & $0,36(0,15-0,57)$ & 0,37 & & & 41,2 \\
\hline \multicolumn{6}{|c|}{ Fenda labial e/ou fenda palatina (Q35-Q37) } \\
\hline Dois dígitos & $0,20(0,05-0,34)$ & 0,28 & 38 & 0,0 & 36,8 \\
\hline Três dígitos & $0,13(0,01-0,24)$ & 0,18 & & & 23,7 \\
\hline \multicolumn{6}{|c|}{ Aparelho digestivo (Q38-Q45) } \\
\hline Dois dígitos & $0,50(0,27-0,74)$ & 0,54 & 18 & 5,6 & 55,6 \\
\hline Três dígitos & $0,40(0,18-0,62)$ & 0,43 & & & 44,4 \\
\hline \multicolumn{6}{|c|}{ Órgãos genitais (Q50-Q56) } \\
\hline Dois dígitos & $0,49(0,30-0,69)$ & 0,75 & 48 & 0,0 & 77,1 \\
\hline Três dígitos & $0,38(0,22-0,55)$ & 0,60 & & & 62,5 \\
\hline \multicolumn{6}{|c|}{ Aparelho urinário (Q60-Q64) } \\
\hline Dois dígitos & $0,51(0,32-0,70)$ & 0,58 & 31 & 0,0 & 61,3 \\
\hline Três dígitos & $0,44(0,27-0,61)$ & 0,49 & & & 51,6 \\
\hline \multicolumn{6}{|c|}{ Aparelho osteomuscular (Q65-Q79) } \\
\hline \multicolumn{6}{|c|}{ Do pé (Q660-Q669) } \\
\hline Dois dígitos & 0,00 & 0,76 & 70 & 1,4 & 81,4 \\
\hline Três dígitos & $-0,01(-0,12-0,11)$ & 0,41 & & & 45,7 \\
\hline \multicolumn{6}{|c|}{ Polidactilia (Q690-Q692/Q699) } \\
\hline Dois dígitos & 0,00 & 0,86 & 113 & 0,0 & 87,6 \\
\hline Três dígitos & $0,15(0,02-0,29)$ & 0,49 & & & 54,0 \\
\hline \multicolumn{6}{|c|}{ Por redução do membro superior (Q710-Q719) } \\
\hline Dois dígitos & 0,00 & 0,43 & 7 & 0,0 & 57,1 \\
\hline Três dígitos & $0,05(-0,20-0,29)$ & 0,02 & & & 14,3 \\
\hline \multicolumn{6}{|c|}{ Não classificadas em outra parte (Q790-Q796) } \\
\hline \multicolumn{6}{|l|}{ Q798-Q799) } \\
\hline Dois dígitos & 0,00 & 0,70 & 55 & 0,0 & 80,0 \\
\hline Três dígitos & $0,68(0,56-0,81)$ & 0,77 & & & 80,0 \\
\hline \multicolumn{6}{|l|}{ Outros } \\
\hline Dois dígitos & $0,37(0,19-0,55)$ & 0,41 & 31 & 0,0 & 45,2 \\
\hline Três dígitos & $0,35(0,19-0,51)$ & 0,36 & & & 38,7 \\
\hline \multicolumn{6}{|c|}{ Anomalias cromossômicas (Q90-Q99) } \\
\hline Dois dígitos & $0,32(0,05-0,60)$ & 0,60 & 20 & 0,0 & 70,0 \\
\hline Três dígitos & $0,37(0,09-0,64)$ & 0,61 & & & 65,0 \\
\hline \multicolumn{6}{|c|}{ Outras malformações congênitas (Q80-Q89) } \\
\hline Dois dígitos & $0,37(0,22-0,52)$ & 0,41 & 37 & 0,0 & 43,2 \\
\hline Três dígitos & $0,25(0,12-0,38)$ & 0,25 & & & 27,0 \\
\hline
\end{tabular}

KS: kappa simples; KAP: kappa ajustado pela prevalência. 
classificados como do sistema osteomuscular $(81,4 \%)$, órgãos genitais $(77,1 \%)$, a anomalias cromossômicas (70\%), aparelho urinário (61,3\%) e digestivo (55,6\%). Quando a análise incluiu o $3^{\circ}$ dígito, somente as anomalias congênitas, órgãos genitais e aparelho urinário apresentaram concordância acima de $50 \%$.

Analisada a confiabilidade por sistemas e aparelhos percebeu-se que os defeitos congênitos classificados como do sistema nervoso central (Q00-Q07), coração e aparelho circulatório (Q20Q28), anomalias cromossômicas (Q90-Q99) e outras malformações congênitas (Q80-Q89) tiveram um kappa simples razoável $(0,21-0,40)$ tanto para 2 como 3 dígitos. Destes apenas as classificações referentes ao coração e aparelho circulatório não passaram a ter kappa ajustado como moderado $(0,41-0,60)$ para 2 ou 3 dígitos. Aparelho digestivo (Q38-Q45), órgãos genitais (Q50-Q56), urinário (Q60-Q64) e osteomuscular (Q65-Q79) obtiveram um kappa ajustado pela prevalência considerado como moderado ou substancial $(0,61-0,80)$ para 2 e 3 dígitos excetuando-se a subcategoria outros do aparelho osteomuscular, avaliada como razoável. Os demais sistemas e aparelhos tiveram suas classificações variando de pobre a razoável.

Optou-se por procederem-se às análises de confiabilidade dos defeitos congênitos listados na Tabela 1 por apresentarem maior freqüência de casos. Percebe-se também que se tratam, além das cromossomopatias, de sistemas e aparelhos cujas anomalias costumam ser detectáveis ao nascimento, exceto os aparelhos digestivo e urinário.

$\mathrm{Na}$ Tabela 2 apresentam-se as medidas de confiabilidade para as informações maternas e do recém-nato, as quais foram consideradas como quase perfeitas $(0,81-1,00)$ excetuando-se a escolaridade, com kappa ajustado pela prevalência de 0,51 (moderado), o número de consultas de pré-natal com kappa de 0,77 (substancial), a duração da gestação com 0,73 (substancial) e o estado civil cujo kappa simples foi de 0,60 (moderado).

Ao analisarem-se os percentuais de concordância entre os prontuários e o SINASC para cada instituição participante da pesquisa, os valores encontrados foram de $25 \%$ a $67 \%$, com média de $44,5 \%$. Tais percentuais variaram muito entre as maternidades, não se encontrando diferenças quando as mesmas foram agrupadas segundo a natureza jurídica da administração.

\section{Discussão}

Nesta pesquisa, tanto as estatísticas kappa quanto os percentuais de concordância mostraram-se bastante variados conforme o tipo de defeito congênito e o grau de especificação pela CID-10. Ficou evidente que as malformações mais complexas e inaparentes ao nascimento mostraram-se com valores de kappa bem menores e com baixos percentuais de concordância sendo o caso das malformações cardíacas, do sistema circulatório e aparelho digestivo. Por outro lado, algumas malformações do sistema músculo esquelético como as do pé, as sindactilias e as polidactilias se mostraram com graus de concordância bem superiores chegando a 87,6\% em análises de 2 dígitos com kappa ajustado quase perfeito.

Todavia, nas malformações do sistema nervoso central classificadas com 2 dígitos da CID-10, verifica-se um kappa ajustado pela prevalência moderado no limite inferior. Para 3 dígitos o kappa ajustado apresentou-se sofrível. Em ambos os estratos os percentuais de concordância estiveram abaixo de $50 \%$. Tais achados se tornam relevantes na medida em que as anomalias do sistema nervoso central, além de muito prevalentes, costumam ser bem evidentes ao nascimento e, portanto mais facilmente descritas.

Estes resultados nos colocam frente a questões relativas ao grau de complexidade e o número de malformações verificadas no recém-nascido ao nascimento. Em estudos com metodologia semelhantes ao presente trabalho os resultados parecem variar conforme o número, tipo e graus de evidência e de complexidade do defeito congênito, sugerindo que tais características influenciam a descrição do defeito congênito no momento de preenchimento das declarações de nascido vivo 25. Portanto, dentre os possíveis motivos para que os relatos dos tipos de defeitos congênitos no SINASC não tenham se mostrado bons nas malformações mais complexas, estaria o fato de muitos destes recém-nascidos receberem diagnósticos imprecisos no período pré-natal assim como no pós-parto imediato, momento reservado para o preenchimento do documento ${ }^{\text {? }}$. Nas instituições em que as declarações de nascido vivo são preenchidas por profissionais não médicos, o acesso ao prontuário para a obtenção de informações pode ser problemático devido à ausência de familiaridade com a terminologia ou ainda porque o diagnóstico da malformação não foi concluído.

Constatou-se neste estudo que o responsável pelo preenchimento das declarações de nascido vivo variou consideravelmente entre as instituições, podendo ser as enfermeiras assistentes das salas de parto, obstetras, pediatras e profissio- 
Medidas de confiabilidade das informações maternas e dos recém-nascidos. Município do Rio de Janeiro, Brasil, 2004.

\begin{tabular}{|c|c|c|c|c|c|c|c|}
\hline Variáveis & $\mathrm{KS}$ & $\mathrm{KP}$ & KAP & Nascidos & $\%$ ignorados & $\%$ Concordantes & $\mathrm{CCl}$ \\
\hline Escolaridade & $0,45(0,38-0,52)$ & $0,51(0,43-0,58)$ & 0,57 & 377 & 42,1 & 65,3 & - \\
\hline Tipo de gravidez & $0,85(0,73-0,96)$ & - & 0,98 & 640 & 1,7 & 98,9 & - \\
\hline Tipo de parto & $0,97(0,95-0,99)$ & - & 0,97 & 636 & 2,3 & 98,4 & - \\
\hline \multicolumn{8}{|l|}{ Número de consultas } \\
\hline de pré-natal & $0,73(0,67-0,78)$ & $0,77(0,72-0,82)$ & 0,76 & 437 & 32,9 & 82,2 & - \\
\hline Apgar no 1ㅇ minuto & $0,96(0,95-0,98)$ & $0,98(0,96-0,99)$ & 0,97 & 627 & 3,7 & 97,0 & - \\
\hline Apgar no 5 o minuto & $0,95(0,93-0,97)$ & $0,97(0,96-0,99)$ & 0,96 & 622 & 4,5 & 96,6 & - \\
\hline Situação conjugal & $0,60(0,53-0,67)$ & - & 0,78 & 457 & 29,8 & 82,5 & - \\
\hline \multicolumn{8}{|l|}{ Número de filhos vivos } \\
\hline em gestações anteriores & $0,86(0,83-0,9)$ & $0,90(0,86-0,93)$ & 0,90 & 511 & 21,5 & 89,8 & - \\
\hline \multicolumn{8}{|l|}{ Número de filhos mortos } \\
\hline em gestações anteriores & $0,60(0,47-0,72)$ & $0,76(0,64-0,88)$ & 0,92 & 448 & 31,2 & 92,7 & - \\
\hline Idade gestacional no parto & $0,68(0,62-0,73)$ & $0,73(0,68-0,78)$ & 0,82 & 592 & 9,1 & 84,4 & - \\
\hline Peso ao nascer & - & - & - & 637 & 14 & - & $0,99(0,99-0,99)$ \\
\hline Idade materna & - & - & - & 646 & 5 & - & $0,98(0,98-0,98)$ \\
\hline
\end{tabular}

KS: kappa simples; KP: kappa ponderado; KAP: kappa ajustado pela prevalência; CCl: coeficiente de correlação intraclasse.

nais de serviços administrativos. Em uma grande maternidade estudada, com mais de 4 mil partos no ano de 2004, todas as declarações são preenchidas no dia seguinte aos partos, por técnicos administrativos. Em uma outra com perfil semelhante os técnicos de enfermagem das salas de parto é que realizam o ofício. Ressalte-se que nestas maternidades os percentuais de concordância entre as fontes analisadas (prontuário/ declarações de nascido vivo) foram respectivamente de 50 e $54 \%$.

Assim, muitas vezes a declaração é liberada sem a descrição do tipo de defeito congênito, com dados incompletos e errados. Parece que quanto mais complexa a malformação, pior a notificação. Logo seria explicável o achado de índices de kappa consideravelmente mais elevados nas análises com 2 dígitos da CID-10 assim como nas malformações de menor complexidade como as do pé e dos órgãos genitais particularmente as hipospádias e as polidactilias.

Sabe-se conforme relatado na literatura que a confiabilidade das informações constantes nas declarações ou certificados de nascimento tem sido avaliada em todo o mundo porque $a$ priori servem como base para o planejamento de políticas de saúde nesta área além de serem amplamente divulgadas nos boletins da maioria dos centros de vigilância epidemiológica. $\mathrm{Nu}$ merosos estudos têm demonstrado que algumas variáveis apresentam graus excelentes de confiabilidade traduzindo a boa coleta destas in- formações e, por conseguinte garantindo maior segurança na sua utilização 6,7,8. Porém, algumas outras variáveis têm-se mostrado problemáticas, alegando-se diversos motivos, desde a falta de uniformização das regras de preenchimento dos dados até o franco desinteresse de quem preenche o formulário $26,27$.

Em uma análise de confiabilidade realizada em alguns hospitais de um estado norte-americano, DiGiuseppe et al. 28 compararam as informações dos certificados de nascimento de mais de 30 mil nascidos vivos com aquelas dos prontuários e demonstraram concordâncias quase perfeitas para variáveis como índice de Apgar, paridade, tipo de parto, tipo de gravidez, idade da mãe dentre outras; mas encontraram resultados de kappa sofríveis para algumas variáveis maternas e da gravidez tais como presença de hipertensão, filho anterior com peso maior que $4.000 \mathrm{~g}$ ou pequeno para a idade gestacional e anomalias fetais.

Também no Brasil, dois estudos relevantes sobre confiabilidade do SINASC realizados no Rio de Janeiro e no Maranhão demonstraram resultados com confiabilidades bem distintas entre as variáveis analisadas. Ao compararem informações de inquéritos epidemiológicos com aquelas de prontuários, Theme Filha et al. 14 encontraram por meio do kappa ajustado pela prevalência, concordâncias quase perfeitas para informações como tipo de parto, tipo de gravidez, índice de Apgar e número de filhos vivos ou mortos em 
gestações anteriores assim como para peso ao nascimento e idade materna através do coeficiente de correlação intraclasse. Por outro lado verificaram-se graus menores de concordância no que se refere à escolaridade materna, número de consultas pré-natais e situação conjugal. Da mesma forma Silva et al. 15 demonstraram um kappa bruto de 0,93 para a variável peso ao nascer e de 0,15 para a idade gestacional no parto.

Tais resultados foram semelhantes aos achados em nossa pesquisa e no que se refere à idade gestacional, isto seria justificável frente à imprecisão no registro desta informação nos prontuários assim como à dificuldade da sua transcrição dentro dos estratos apresentados nas declarações de nascido vivo. Em tais situações, é necessário o maior empenho do responsável pelo preenchimento da declaração de nascido vivo na obtenção e transcrição correta do dado.

Embora possa ser questionada a escolha de prontuários para fins de comparação com os dados das declarações de nascido vivo ${ }^{29}$, tentamos contornar possíveis vieses procedendo à pesquisa exaustiva dos mesmos com médicos especialistas em malformações congênitas para que todos os defeitos congênitos descritos fossem relatados nos formulários de coleta e posteriormente comparados. Verificou-se também que na maioria das vezes os relatos sobre defeitos congênitos nos prontuários foram realizados por médicos assistentes na sala de parto, obstetras e pediatras, ou nos casos que demandaram internação prolongada, pelos neonatologistas ou outros especialistas. O fato de 29 casos com defeitos congênitos segundo o SINASC não terem sido confirmados após pesquisa nos prontuário levanta a hipótese de que houve erro ao se preencher o campo 34 da declaração de nascido vivo, tendo em vista não ter sido encontrado transcrito em nenhuma delas qualquer tipo de defeito congênito.

Outra importante questão diz respeito à codificação do defeito congênito relatado na declaração de nascido vivo. A descentralização do SINASC no Município do Rio de Janeiro deu-se de forma paulatina a partir de 2003 sendo que atualmente todas as declarações de nascido vivo são digitadas nas CAP, ou seja, fora da SMS-RJ. O fato de somente um único diagnóstico de defeito congênito poder ser escolhido para codificação na declaração de nascido vivo obriga que o codificador faça uso de algum critério de seleção nos casos com malformações múltiplas. Na falta de critérios precisos, o código da CID-10 a ser escolhido dependerá de particularidades que podem não permitir a generalização das informações e a posterior comparação das mesmas dentro e fora do município.

Por fim, vale ressaltar que apesar das limitações, os resultados ora encontrados apontam para importantes questões relacionadas à notificação de defeito congênito pelo SINASC. Primeiramente, que o campo 34 poderia ser mais bem explorado para informações sobre defeitos congênitos em nossa população, não só porque estas anomalias estão entre as primeiras causas de mortalidade na infância, mas também porque não haveria até o momento outra fonte melhor de informação sobre o tema, com a abrangência do SINASC.

Também parecem necessários investimentos na qualificação da informação, seja no pessoal envolvido nesta tarefa, seja através de recomendações sobre o preenchimento das declarações de nascido vivo em casos de defeitos congênitos, o que resultaria em maior empenho na transcrição do eventual diagnóstico realizado pelos médicos. Além disso, seria de grande valia a elaboração de critérios para a codificação dos defeitos congênitos transcritos nas declarações de nascido vivo, visto que não se sabe qual o defeito congênito a ser escolhido pelo codificador em casos de recém-nascidos com múltiplas malformações.

Este estudo revela apenas parte dos problemas relacionados à qualidade das informações sobre defeitos congênitos no Município do Rio de Janeiro, portanto faz-se necessária a realização de estudos mais abrangentes e aprofundados versando sobre o universo particular de cada maternidade assim como o processo de codificação do campo 34 realizado pela SMS-RJ. 


\section{Resumo}

Avaliou-se a confiabilidade das informações sobre defeitos congênitos contidas nas declarações de nascido vivo do Sistema de Informações sobre Nascidos Vivos (SINASC) no Município do Rio de Janeiro, Brasil, no ano de 2004 comparando-as com os prontuários hospitalares destas crianças e de suas mães na internação para o parto. Após a seleção de 24 maternidades do SUS, os tipos de defeitos congênitos constantes nos prontuários foram transcritos em formulários de coleta próprios, codificados com base na CID-10 e comparados com os arquivos do SINASC. Verificou-se uma maior freqüência de defeitos congênitos dos sistemas osteomuscular e nervoso central e percentual de concordância acima de 50\% nos aparelhos digestivo, urinário e osteomuscular, órgãos genitais e de anomalias cromossômicas. O kappa ajustado pela prevalência variou conforme as análises para 2 ou 3 dígitos da CID-10, com melhores resultados nos aparelhos osteomuscular, genito-urinário, digestivo e as anomalias cromossômicas e os piores nos sistemas nervoso central e cárdio-circulatório, malformações congênitas da fa$c e$, olhos pescoço e orelhas e fendas lábio-palatinas. Os resultados encontrados são insatisfatórios e apontam para a necessidade de qualificação do pessoal envolvido no preenchimento das declarações assim como a padronização da codificação dos defeitos congênitos.

Anormalidades; Declaração de Nascimento; Sistemas de Informação

\section{Referências}

1. Rosano A, Botto LD, Botting B, Mastroiacovo P. Infant mortality and congenital anomalies from 1950 to 1994: an international perspective. J Epidemiol Community Health 2000; 54:660-6.

2. Penchaszadeh VB. Implementing comprehensive genetic services in developing countries: Latin America. Birth Defects Orig Artic Ser 1992; 28: 17-26.

3. Penchaszadeh VB. Establecimiento de servicios integrales de genética en los países en desarrollo: América Latina. Bol Oficina Sanit Panam 1993; 115:39-47.

4. Horovitz, DDG, Llerena Jr. JC, Mattos RA. Atenção aos defeitos congênitos no Brasil: panorama atual. Cad Saúde Pública 2005; 21:1055-64.

\section{Colaboradores}

F. A. R. Guerra foi responsável pela análise e interpretação dos dados e redação do artigo. J. C. Llerena Jr. e S. G. N. Gama colaboraram na orientação e revisão. C. B. Cunha foi responsável pela construção dos bancos e análise dos dados. M. M. Theme Filha colaborou na análise dos dados e redação.

\section{Agradecimentos}

À Secretaria Municipal de Saúde do Rio de Janeiro.
5. Botto LD, Mastroiacovo P. Surveillance for birth defects and genetics disease. In: Khoury MJ, Burke W, Thomson EJ, editors. Genetics and public health in 21 st century. Oxford: Oxford University Press; 2000. p. 123-40.

6. Piper JM, Mitchel Jr. EF, Snowden M, Hall C, Adams M, Taylor P. Validation of 1989 Tennessee birth certificates using maternal and newborn hospital records. Am J Epidemiol 1993; 137:758-68.

7. Watkins ML, Edmonds L, McClearn A, Mullins L, Mulinare J, Khoury M. The surveillance of birth defects: the usefulness of the revised US standard birth certificate. Am J Public Health 1996; 86: 731-4.

8. Kirby RS. The quality of data reported on birth certificates. Am J Public Health 1997; 87:301. 
9. Mello-Jorge MHP, Gotlieb SLD, Soboll MLMS, Almeida MF, Latorre MRDO. Avaliação do Sistema de Informação sobre Nascidos Vivos e o uso de seus dados em epidemiologia a estatísticas de saúde. Rev Saúde Pública 1993; 27:2-4.

10. Silva RI, Theme Filha MM, Noronha CP. Sistema de Informação sobre Nascidos Vivos na cidade do Rio de Janeiro. Inf Epidemiol SUS 1997; 2:33-48.

11. Maia MAC. Caracterização dos nascidos vivos hospitalares no primeiro ano de implantação do Subsistema de Informação sobre Nascidos Vivos, em município de Minas Gerais. Rev Saúde Pública 1997; 31:581-5.

12. Rodrigues CS, Magalhães Jr. HM, Evangelista PA, Ladeira RM, Laudares S. Perfil dos nascidos vivos no Município de Belo Horizonte, 1992-1994. Cad Saúde Pública 1997; 13:53-7.

13. Helena ETS, Wisbeck J. Implantação do SINASC e perfil dos nascidos vivos de Blumenau, 1994-1997. Inf Epidemiol SUS 1998; 7:35-42.

14. Theme Filha MM, Gama SGN, Cunha CB, Leal MC. Confiabilidade do Sistema de Informações sobre Nascidos Vivos Hospitalares no Município do Rio de Janeiro, 1999-2001. Cad Saúde Pública 2004; 20 Suppl 1:S83-91.

15. Silva AAM, Ribeiro VS, Borba Jr. AF, Coimbra LC, Silva RA. Avaliação da qualidade dos dados do Sistema de Informação sobre Nascidos Vivos em 1997-1998. Rev Saúde Pública 2001; 35:508-14.

16. Honein MA, Paulozzi J. Birth defects surveillance: assessing the "gold standard". Am J Public Health 1999; 89:1238-40.

17. Wen SW, Rouleau J, Lowry RB, Kinakin B, Anderson-Redick S, Sibbald B, et al. Congenital anomalies ascertained by two record systems run in parallel in the Canadian Province of Alberta. Can J Public Health 2000; 91:193-6.

18. Boyd PA, Armstrong A, Dolk H, Botting B, Pattenden S, Abramsky L, et al. Birth defects surveillance in England: ascertainment deficiencies in the national system. BMJ 2005; 330:27.
19. Organização Mundial da Saúde/Centro Colaborador da OMS para a Classificação de Doenças em Português, Universidade de São Paulo. Classificação estatística internacional de doenças e problemas relacionados à saúde - CID-10. São Paulo: Edusp; 1994.

20. Baskett TF, Naegele F. Naegele's rule: a reappraisal. BJOG 2000; 107:1433-5.

21. Byrt T, Bishop J, Carlin JB. Bias, prevalence and kappa. J Clin Epidemiol 1993; 46:423-9.

22. Sim J, Wright CC. The kappa statistic in reliability studies: use, interpretation, and sample size requirements. Phys Ther 2005; 85:257-68.

23. Landis JR, Koch GG. The measurement of observer agreement for categorical data. Biometrics 1977; 33:159-74.

24. Abramson JH. WINPEPI (PEPI-for-Windows) computer programs for epidemiologists. Epidemiol Perspect Innov 2004; 1:6.

25. Snell LM, Little BB, Knoll KA, Johnston Jr. WL, Rosenfeld CR, Gant NF. Reliability of birth certificate reporting of congenital anomalies. Am J Perinatol 1992; 9:219-22.

26. Roohan PJ, Josberger RE, Acar J, Dabir P, Feder HM, Gagliano PJ. Validation of birth certificate data in New York State. J Community Health 2003; 28:335-46.

27. Northan S, Polancich S, Restrepo E. Birth certificate methods in five hospitals. Public Health Nurs 2003; 20:318-27.

28. Di Giuseppe DL, Aron DC, Ranbom L, Harper DL, Rosenthal GE. Reliability of birth certificate data: a multi-hospital comparison to medical records information. Matern Child Health J 2002; 6:169-79.

29. Veras CMT, Martins MSA. Confiabilidade dos dados nos formulários de autorização de internação hospitalar (AIH), Rio de Janeiro, Brasil. Cad Saúde Pública 1994; 10:339-55.

Recebido em 07/Fev/2006

Versão final reapresentada em 28/Fev/2007 Aprovado em 24/Jul/2007 